\title{
CONSIDERACIONES SEMÁNTICAS ACERCA DEL USO DEL DATIVO ALEMAN Y SUS POSIBLES CONCURRENCIAS CON OTRO TIPO DE CONSTRUCCIONES.
}

\author{
Rafael López-Campos Bodineau
}

Una de las perspectivas más interesantes a la hora de abordar un estudio acerca del dativo 'alemán son los distintos tipos de frases preposicionales con las que éste puede concurrir. Nuestro objetivo en este sentido es establecer una clara distinción entre los diversos tipos de dativo y observar del mismo modo con qué tipo de estructuras se puede identificar cada uno.

Partamos para ello del principio básico de la gramática de dependencia, desarrollada fundamentalmente por L. Tesnière con su obra Grundzüge der strukturalen Syntax: todo elemento oracional tiene una relación de dependencia respecto al verbo en la oración. Es lo que se denomina relación Regens - Dependens, que Tesnière toma como fundamento de su teoría. El verbo tiene siempre la función de Regens y cualquiera de los restantes elementos oracionales la de Dependens. De este modo cada verbo o Regens tendrá un número determinado de los denominados Dependens, todo en relación con el contexto y el sentido de la oración ${ }^{1}$.

Tesnière afirma también de modo genérico que no todos los elementos oracionales tienen por qué tener el mismo tipo de relación respecto al nucleo verbal, si atendemos a la relevancia semántica de dichos elementos. Por ejemplo, una frase nominal con función sintáctica de objeto directo que esté regida obligatoriamente por el núcleo verbal no tiene el mismo valor semántico que un adverbio con carácter facultativo en una oración. De este modo y en lo que concierne al dativo, podemos afirmar que éste, dependiendo del caso, puede tener mayor o menor importancia semántica y de este modo podemos establecer dos tipos fundamentales de dativo: un dativo obligatorio, en los casos en los que la presencia de éste es imprescindible para que la oración tenga un sentido completo, y otro dativo que puede

\footnotetext{
1 Tesnière propone un sistema jerárquico de relacion entre todos los elementos oracionales. De este modo hemos de hacer la salvedad que el verbo no sería el único en poder desempeñar esta función de Regens. En una oración del tipo er hat das Auto deines Vaters gesehen el complemento del nombre en genitivo deines Vaters es Dependens respecto al Regens das Auto, que a su vez es Dependens respecto al que consideramos Regens por antonomasia, es decir el núcleo verbal..
}

Tesnière, Lucien. Grundzüge der strukturalen Syntax. Klett-Cotta. Stuttgart. 1980. Pg. 28. 
incluso tener un uso facultativo por la poca relevancia que tiene, desde el punto de vista de la información 2 : es el que llamamos dativo libre o freier Dativ.

Desde un punto de vista estrictamente sintáctico, por lo tanto, podemos afirmar que en ambos casos la presencia del dativo viene dada por el régimen verbal, pero desde un punto de vista semántico tendremos que hacer dos observaciones:

- El dativo podrá tener un uso obligatorio o libre, dependiendo de la carga semántica del dativo en cada uno de los casos.

- En segundo lugar, tendremos que distinguir entre los distintos valores semánticos que puede aportar el dativo.

Como ejemplos de dativo obligatorio podemos mencionar cualquiera de los que Schumacher, Engel u otros nos recogen en sus diccionarios de valencias como verbos con los que es característico un régimen de dativo:

jdm. etwas geben, jdm. etwas schicken, etwas gehört jdm.

En todos ellos la ausencia del dativo cambiaría o incluso dejaría completamente sin sentido a la oración, lo cual no ocurre en los casos en los que aparece un dativo de los que denominamos libre.

En un número considerable de casos una frase nominal en dativo puede concurrir con una frase preposicional, cuya preposición introductoria depende del tipo de valor semántico que tenga cada dativo. Para ello pasaremos a analizar cada uno de los tipos de dativo en lengua alemana, los distintos valores semánticos que cada uno de esos tipos puede tener y, en consecuencia, el tipo concurrencia que se puede dar en cada uno de ellos.

En primer lugar hagamos una breve mención a los seis tipos de dativo que contempla la gramática alemana y en los que queremos centrar nuestra atención:

1. Dativo commodi: Este dativo tiene el valor semántico de ser el beneficiario de la acción verbal. Obsérvese:

jdm. etwas vorschlagen,

jdm. von etwas berichten.

2. Dativo incommodi: se caracteriza por tener un valor semántico opuesto al anterior, es decir, el de ser el perjudicado por la acción verbal:

jdm. eine Bitte abschlagen,

der Bleistift ist mir heruntergefallen.

\footnotetext{
2 Helbig y Buscha, al tratarlos, consideran al primero como Hauptsatzglied o parte integrante fundamental de la oración y al segundo como sekundäres Satzglied o elemento secundario de la oración. 1989. Pg. 552.

Helbig, Gerhard y Buscha, Joachim. Deutsche Grammatik. VEB Verlag Enzyklopädie. Leipzig.
} 
3. Dativo de medida (Dativ des Maßstabs): tiene el valor semántico de expresar una consideración subjetiva en la persona a la que se refiere el dativo:

das fällt mir schwer, die Zeit vergeht mir schnell.

4. Dativo de juicio o Dativ des Zustandsträgers: se caracteriza por ser un dativo con el valor semántico de expresar la impresión de algo referido a alguien:

das ist mir ein Rätsel, das ist mir ein Trost.

5. Dativo simpatético o possessiver Dativ: es el dativo que compite con el pronombre posesivo y, por lo tanto, comparte con él el mismo valor semántico:

ich habe mir die Hände gewaschen,

*ich habe meine Hände gewaschen.

6. Dativo ético o ethischer Dativ: muchos autores lo denominan también dativo de interés ya que su valor semántico es el de expresar un interés por parte de la persona en dativo:

mach mir die Tür nicht kaputt!

Cada uno de estos dativos se puede comportar de modo libre u obligatorio en la oración, si bien podemos afirmar que los últimos cuatro tipos de dativo expuestos tienen en la totalidad de los casos un uso libre, es decir, semánticamente tienen una relevancia secundaria y su uso es facultativo en la oración.

Antes de entrar con detalle en cada uno de los tipos mencionados debemos hacer referencia a que en muchos casos una forma en dativo puede enmarcarse en el ámbito semántico de más de uno de ellos. Oraciones como:

mach mir das Fenster zu! kauf mir einen Bleistift!

pueden identificarse con un dativo ético, con un dativo commodi, o incluso con ambas cosas, dependiendo de la intención del hablante y, en definitiva, del contexto de la oración ${ }^{3}$.

Dentro de los DATIVOS COMMODI E INCOMMODI, podemos afirmar que hay un gran número de casos en los que éstos tienen un uso obligatorio, ya que la presencia de cualquiera de los dos da en un gran número de casos un sentido completo a la oración y al verbo que lo introduce. Obsérvense los siguientes ejemplos:

jdm. zulächeln,

jdm. etwas schenken,

jdm. etwas schicken,

jdm. etwas zeigen,

3 Agel, Vilmos. Überlegungen zur Theorie und Methode der historisch-synchronen Valenzsyntax und Valenzlexikographie. Niemeyer. Tübingen. 1988. Pgs. 117-123. 
jdm. antworten, jdm. etwas ablehnen,

etwas ist mir gebrochen.

Éstos son todos ejemplos de lo que en alemán se denominan verbos con los que es característico un régimen de dativo, es decir, desde un punto de vista sintáctico todos estos verbos van acompañados siempre de una frase nominal en dativo. Semánticamente podemos considerarlos como ejemplos de dativo commodi o incommodi, según tengan el valor semántico de ser el beneficiario o el perjudicado de la acción verbal y, por otro lado, podemos atribuírle un uso obligatorio, ya que la presencia de todos ellos es imprescindible para que la oración tenga un sentido completo. Oraciones como

*er hat zugelächelt,

*er hat das Auto geschenkt.

resultan incompletas e incorrectas, ya que falta una frase nominal en dativo, que es lo que les da un sentido completo.

En el contexto de los dativos commodi o incommodi con uso obligatorio es posible establecer cuatro concurrencias:

1. En primer lugar es siempre es posible la concurrencia entre un dativo commodi y una frase preposicional con $f u ̈ r^{4}$. En ese caso el dativo tiene el valor semántico más genérico de ser el beneficiario de la acción. Obsérvense los siguientes ejemplos:

das Angebot eröffnet mir neue Möglichkeiten,

das Angebot eröffnet neue Möglichkeiten für mich, ${ }^{5}$

er setzt seinem Freund ein Ziel,

er setzt ein Ziel für seinen Freund.

Debido al sentido que tiene ya de por sí la preposición für podemos afirmar que esta concurrencia no se da en los ejemplos de dativo incommodi. Obsérvese el siguiente ejemplo:

die Vase ist mir gebrochen,

* die Vase ist für mich gebrochen.

2. También es frecuente la concurrencia entre un dativo commodi o incommodi y una frase preposicional con an + acusativo, en los casos en que el dativo adopta el valor semántico de ser el receptor de algo. Esto ocurre con los que Josef Schmid denomina Verben des Gebens:

jdm. etwas schreiben, etwas an jdn. schreiben,

\footnotetext{
4 Schmid, Josef. Zum Verhältnis von Dativ und Präpositionalphrase mit für im heutigen Deutsch. En Sprachwissenschaft. Vol. 6. Carl Winter-Universitätsverlag. Heidelberg. 1981. Pg. 152.

5 Drosdowski, Günther. Das große Wörterbuch der deutschen Sprache. Tomo 2. Bibliographisches Institut Mannheim. Duden Verlag. Mannheim. 1976. Pag. 741.
} 
jdm. etwas überweisen, etwas an jdn. überweisen,

jdm. etwas verabreichen, an jin. etwas verabreichen. ${ }^{6}$

3. Matzel también introduce otros casos de preposiciones en los que se da esta identificación entre dativo y frase preposicioinal. Nos referimos a los casos de las preposiciones in, mit $\mathrm{u}$ otras en ejemplos como:

das Fach gliedert sich den Geisteswissenschaften ein,

das Fach gliedert sich in die Geisteswissenschaften ein,

das wäre einer Katastrophe gleichzusetzen, das wäre mit einer Kathastrophe gleichzusetzen?

4. Por último resultan especialmente relevantes algunas identificaciones entre construcciones con dativo commodi o incommodi y frases preposicionales introducidas por an + acusativo en el contexto de verbos que a su vez están compuestos por el prefijo verbal an-. En estos casos la frase en dativo tiene el valor semántico de expresar un acercamiento o aproximación:

sich einer Sache anschliessen,

sich an eine Sache anschliessen,

sich jdm. anhängen,

sich an jdn. anhängen.

Al hablar del dativo en general muchos autores hacen una referencia especial a la construcción denominada Dativpassiv o bekommen-passiv, la cual se forma con los verbos bekommen, erhalten o kriegen y el participio II alemán ${ }^{8}$. Esta construcción ha sido siempre identificada y estudiada con el dativo porque es resultado de dos construcciones en dicho caso:

sie erkennen mir das Studium in Deutschland an

$\mathrm{y}$

mir wird das Studium in Deutschland anerkannt,

de la que resulta una de estas oraciones que denominamos como Dativpassiv:

mein Studium in Deutschland habe ich anerkannt bekommen.

\footnotetext{
6 Engel, Ulrich und Schumacher, Helmut. Kleines Valenzlexikon. Gunter Narr Verlag. Tübingen. 1978. Pag. 278 .

7 Matzel, Klaus. Dativ und Präpositionalphrase. En Sprachwissenschaft. Vol. 1. Carl Winter-Universitätsverlag. Heidelberg. 1976. Pag. 144.

8 Eroms, Hans-Werner. Zur Konversion der Dativphrasen. En Sprachwissenschaft. Vol. 2. Carl WinterUniversitätsverlag. Heidelberg. 1978. Pg. 386.
} 
A esta construcción hemos de referirnos obligatoriamente al hablar de concurrencias entre el dativo commodi o incommodi y otras construcciones. Eroms establece, de este modo, una relación de los verbos de dativo que admiten esta construcción o, al menos, con los que es frecuente. Obsérvense la siguientes identificaciones:

das wurde mir anerkannt,

das habe ich anerkannt bekommen,

das wurde mir zugeschickt,

das habe ich zugeschickt bekommen,

sie haben mir den Ball zugeworfen,

den Ball habe ich zugeworfen bekommen.

Hasta ahora nos hemos referido tan sólo a casos en los que el uso del dativo es obligatorio para que la oración forme un sentido completo, pero por otro lado tenemos ejemplos de dativo commodi o incommodi en los que éste no tiene semánticamente una relevancia tan importante en la oración y, por lo tanto tienen un uso libre.

Como ejemplos podemos mencionar:

jdm. die Tür öffnen,

das ist mir ein Trost.

en los que la verdadera carga semántica de la oración no está en el dativo, sino en los elementos de la oración que vienen dados por la valencia verbal. En el ejemplo jdm. die Tür öffnen, la valencia verbal rige obligatoriamente un acusativo etwas öffnen y el uso del dativo es meramente facultativo con la única función de enfatizar la referencia al sujeto hacia el que va dirigido la acción. Lógicamente hay un gran número de verbos en los que el dativo se puede considerar libre o no, dependiendo del valor oracional que se quiera enfatizar y fundamentalmete del contexto pragmático en que aparezca. Observemos otro ejemplo:

er hat ihm einen Kaffee gekocht

En él observamos que el dativo ihm puede ser libre o no, dependiendo del elemento oracional que se quiera enfatizar. El verbo kochen se considera normalmente bivalente, es dedir jd. kocht etwas y en este sentido se puede afirmar que al admitir un dativo se trata de uno de estos que denominamos libre. Pero observemos el siguiente contexto oracional:

Wem hat er den Kaffee gekocht? seinem Vater?

Nein, seinem Bruder (hat er einen Kaffee gekocht).

En este contexto se aprecia que el dativo que en principio consideramos libre o con carácter facultativo en el contexto oracional también se puede concebir como obligatorio, ya que en este contexto se quiere enfatizar el objeto indirecto como parte principal de la oración.

A la hora de hablar de los dativos commodi e incommodi con uso libre en la oración podemos destacar dos construcciones fundamentales con las que puede concurrir: 
1. En primer lugar, y del mismo modo que con el dativo obligatorio, existe una clara identificación con la mencionada construcción de Dativpassiv. Obsérvense los siguientes ejemplos:

er baut ihm ein Haus,

er bekommt ein Haus gebaut,

Otto, mach mir bitte das Fenster zu,

Ja du bekommst es zugemacht.

En todos estos ejemplos se observa que el dativo tiene semánticamente un valor facultativo, ya que se podría prescindir de él, sin que por ello cambie el sentido de la oración. Obsérvese:

er baut ein Haus,

Otto, mach bitte das Fenster zu.

2. También se da en la mayoría de los casos una identificación total entre el dativo commodi y una frase preposicional con für, sin que por ello cambie el sentido de la oración. Obsérvense los siguientes ejemplos:

Mach die Tür auf für ihn!

Sie wollte ihm die Tür nicht aufmachen.

Existe un número de verbos en los que el dativo tendrá un valor semántico u otro, dependiendo de la frase preposicional con que pueda ser identificado. Obsérvese de este modo que la oración

jdm. etwas schreiben,

puede ser identificada con

etwas für jdn. schreiben,

etwas an jdn. schreiben.

En ambos casos el dativo tiene un uso libre en la oración, ya que podemos prescindir de él, pero el sentido de la oración estará determinado por la frase preposicional con la que podamos identificar al dativo? .

El dativo de juicio o DATIV DES ZUSTANDSTRÄGERS se caracteriza por establecer una referencia entre un sustantivo y el sujeto de una acción. Hay en este caso, por lo tanto, un componente morfológico muy importante que es la presencia de un sustantivo como introductor del objeto indirecto ${ }^{10}$ :

${ }^{9}$ Latour, Bernd. Verbvalenz. Eine Einführung in die dependentielle Satzanalyse des Deutschen. Hueber. München. 1985. Pgs. 55-59.

10 Algunos autores como Josef Schmid incluyen este dativo dentro del apartado general de los dativos que pueden ser introducidos por un sustantivo. Schmid afirma que este dativo se distingue de los demás en la capacidad que tiene de ser introducido por un sustantivo y sostiene la presencia del dativo implica una tendencia a dar un matiz positivo en relación, por ejemplo, con una frase preposicional con für, la cual tiene una tendencia 
es ist mir ein Rätsel, es ist mir ein Trost.

Este tipo de dativo se caracteriza por ser equivalente en la totalidad de los casos a una frase preposicional con für + acusativo.

De este modo podremos formular también:

das ist ein Rätsel für mich, das ist ein Trost für mich.

El dativo de medida, también denominado dativus iudicantis o DATIV DES $M A \beta S T A B S$ se caracteriza por tener una implicación subjetiva desde la perspectiva del sujeto y morfologicamente viene dado por la presencia siempre de un adjetivo que introduce el dativo.

Obsérvese:

die Zeit vergeht mir schnell, das Buch ist mir zu schwer.

De este modo el dativo va siempre en estos casos unido a adjetivos, pero es importante distinguir que no se puede aplicar a cualquiera de ellos. En la mayoría de los casos habremos de utilizar una construcción ich finde es + adj. Josef Schmid, que denomina a este dativo Dativ des Standpunktes, afirma que existe un grupo muy concreto de adjetivos que son los que admiten un régimen directo en dativo ${ }^{11}$. En los demás casos o bien hay que recurrir a la mencionada construcción con el verbo finden, o bien utilizar una frase preposicional introducida por für. El adjetivo interessant, por ejemplo no admite una frase nominal en dativo y entonces tendremos:

* das ist mir interesant, das finde ich interessant, das ist interessant für mich

Josef Schmid, en este sentido, establece a su vez algunas diferencias de tipo semántico entre las construcciones de dativo y la de für con acusativo. Obsérvense los ejemplos:

a dar un matiz de negativo. De este modo afirma que hay sustantivos con los que hay una tendencia mayor a acepatar el dativo, ya que tienen un sentido positivo, por ejemplo Ansporn, ehre, Erlebnis, Freude, Hilfe, Lehre, etc, y por otro, que la mayoría de los sustantivos con un sentido negativo tienen por lo tanto la tendencia a admitir preferentemente la frase preposicional con für. Tales son Bedrohung, Behinderung, y otros. Pero recalcamos que ambas construccciones son válidas en el alemán actual.

Schmid, Josef. Zum Verhältnis von Dativ und Präpositionalphrase mit für im heutigen Deutsch. En Sprachwissenschaft Vol. 6. Carl Winter Universitätsverlag. Heidelberg. 1981. Pg. 158.

11 Buscha, en este sentido, recoge algunos como gleichgültig, fremd, hinderlich, nützlich, bekannt, unerklärlich, etc. como adjetivos que introducen necesariamente una construcción con dativo o Dativ des Maßstabs:

diese Stadt ist mir bekannt,

es ist mir unerklärlich, warum du das Auto nicht gekauft hast,

etc.

Buscha A. und Buscha B. Deutsches Übungsbuch. VEB Verlag Enzyklopädie Leipzig. 1990. Pg. 205. 
das ist mir zu schwer,

das ist sehr schwer für mich.

El primer ejemplo se trata de un Dativ des Maßstabs, y con él queda expresado el carácter subjetivo de la acción. El segundo, por contrario, hace alusión a un planteamiento objetivo de la realidad referido al sujeto de la acción verbal.

En lo que se refiere al DATIVO SIMPATÉTICO o possesiver Dativ, debemos destacar que la concurrencia más importante es la que tan sólo en algunos casos se da con construcciones de acusativo o bien de adjetivo posesivo, aunque debemos subrayar que en estos casos siempre se da un cambio de sentido, aunque sea muy leve, al utilizar una u otra.

El aspecto posesivo de una acción verbal o bien la implicación de un sujeto activo en dicha acción verbal se puede expresar en alemán de tres modos distintos: mediante un possessiver Dativ, un acusativo o bien una construcción de adjetivo posesivo y es debido a la capacidad de concurrencia que tienen estas tres construcciones entre sí por lo que vamos a estudiarlas con la misma atención. Observemos en primer lugar algunos ejemplos con construcción de possessiver Dativ:

sich die Fingernägel abschneiden od. abbrechen,

sich ein Bein brechen,

sich die Zähne putzen,

jdm. auf die Schulter tippen,

jdm. auf die Schulter klopfen,

mir klopft das Herz,

mir schmerzt der Kopf,

er schlägt seinem Gegner die Nase,

der Arzt klopft mir den Rücken ab,

mir knurrt der Magen vor Hunger,

es knackt mir in allen Gelenken,

jdm. die Hand schütteln.

Todos estas oraciones responden a ejemplos típicos de possessiver Dativ. En todos ellos es imprescindible el uso de dativo para expresar la referencia al sujeto de la acción y en este caso no son admisibles concurrencias con acusativo del tipo de:

*ich schneide mich die Fingernägel $a b$,

$*_{i c h}$ habe mich das Bein gebrochen ${ }^{12}$.

\footnotetext{
12 Recordemos que la lengua alemana tan sólo admite un doble acusativo con el uso de verbos muy concretos como fragen, abfragen, hinabstürzen y lehren en construcciones como:

das habe ich dich nicht gefragt,

der Lehrer hat seine Schüler zwei Abschnitte abgefragt,

er hat den Stein den Berg hinabgestürzt, etc.

o con expresiones de tiempo del tipo

er hat den ganzen Tag Deutsch gesprochen.
} 
Tenemos que afirmar que en muchos de los casos sí son admisibles concurrencias del possessiver Dativ con construcciones en las que el sujeto de la acción vaya expresado mediante un adjetivo posesivo. De este modo obsérvense los siguientes pares de oraciones:

(a) ich habe mir die Fingernägel abgebrochen,

(b) ich habe meine Fingernägel abgebrochen,

(a) ich habe mir das Bein gebrochen,

(b) ich habe mein Bein gebrochen.

Lo que ocurre es que en todos estos casos hay un cambio de sentido en cada una de las oraciones. En las que están marcadas con (a) no se expresa intencionalidad en la acción, mientras que en las que están marcadas con (b) sí hay una clara voluntad por parte del sujeto de llevar a cabo la acción verbal.

Por otro lado y en segundo lugar debemos hacer referencia a verbos, cuya expresión solo es posible mediante una construcción de adjetivo posesivo. Obsérvese:

mein Blutdruck ist gefallen od. gestiegen,

meine wunde brennt,

mein Finger ist geschwollen,

mein Kopf tut weh.

En ellas es imposible la concurrencia con cualquiera de las otras dos construcciones de dativo o acusativo:

*der Blutdruck ist mir gefallen,

*der Kopf tut mir weh,

*der Kopf tut mich weh.

Finalmente existe un grupo muy amplio de verbos que llevan forzosamente una construcción de acusativo. Obsérverse:

Mich juckt es am Arm,

sich am Arm kratzen,

verbessere mich!

Korrigiere mich!

jdn. in den Arm beißen,

$j d n$. ins rechte ohr sprechen,

mich nervt,

mich macht das nicht an.

En este caso sólo es posible establecer concurrencias con adjetivo posesivo en algunos de los ejemplos. De este modo:

verbessere meine Fehler!

verbessere mich!

korrigiere meine Fehler!

korrigeire mich! 
En resumen, cada uno de estos tres grupos de construcciones hacen referencia a acciones verbales muy análogas desde el punto de vista semántico, la diferencia está en que en unas se ha impuesto la forma en acusativo, en otras la del pronombre poseesivo y en otras por último la de dativo ${ }^{13}$.

De todos modos tenemos que destacar que hasta la edición de las primeras gramáticas y la formalización de la lengua alemana en el siglo XIX no había una fijación tan estricta acerca del uso de una u otra de estas tres construcciones, es decir, la posibilidad de concurrencia era mayor entre las tres. Analicemos, por ejemplo, los verbos fallen o steigen. Cualquiera de ellos aparece normalmente formulado con la construcción de dativo. Obsérvense, de este modo, ejemplos como:

das Buch ist ihm aus der Hand gefallen, die Haare fallen ihm strähnig ins Gesicht,

die Stelle ist ihr in den Kopf gestiegen, der Duft steigt mir in die Nase.

En todo caso, y con otro sentido, puede aparecer con la construcción de adjetivo posesivo en ejemplos como:

mein blutdruck ist gestiegen od. gefallen.

Pues bien, los Hermanos Grimm recogen en muchos casos un uso indistinto de ambas construcciones en la lengua hablada del siglo XVI y de este modo hacen referencia a una completa identificación semántica entre oraciones como:

er ist im zu füszen gefallen, er ist im zu seinen füszen gefallen ${ }^{14}$.

Lo que es imposible es la concurrencia de más de una de ellas, como por ejemplo:

*korrigiere mich meine Fehler,

*mein Kopf tut mir weh.

Muchos autores denominan a este tipo de dativo Pertinenzdativ. También hemos de destacar que la sustitución por la construcción de Dativpassiv es sólo posible en algunos casos. Obsérvese que de este modo Eroms acepta ejemplos como:

ihm wurde der letzte Wunsch erfüllt, er bekommt seinen letzten Wunsch erfüllt,

pero no acepta esta transformación en oraciones como

der Schlüssel ist mir ins Wasser gefallen.

13 Behagel, O. Beiträge zur deutschen Syntax (Vertauschung von Genitiv, Dativ, Akkusativ beim persönlichen Pronomen). En Germania Her. von Pfeifer. 1879. Verlag der J. B. Metzer’schen Buchhandlung. Stuttgart, 1879. Pgs. 24-46.

14 Grimm, Jacob und Wilhelm. Deutsches Wörterbuch. dTV. München. 1984. Tomo 3. Pg. 1281. 
La marca de la persona debe por lo tanto aparecer tan sólo una vez en el conjunto de contexto oracional.

Al hablar de este tipo de dativo debemos también hacer referencia a un fenómeno muy interesante, que es el cambio de sentido que se produce en muchos de estos casos al usar el dativo o el acusativo. Los verbos beißen, boxen, kneifen, küssen, puffen, schlagen, schneiden, stechen, zwicken pueden regir dativo o acusativo.

Si aparecen con la construcción de acusativo el receptor de la acción es lo importante y se da a entender que dicha acción se ha llevado a cabo en su integridad:

\section{Er kneift ihn auf den Arm.}

Pero si aparecen con la fórmula de acusativo lo que se pone de relieve es la intención del sujeto de llevar a cabo la acción verbal, sin importar si se realiza o no:

Er kneift ihm auf den Arm.

El possessiver Dativ se caracteriza también por no ser sustituíble en ninguno de los casos por frase preposicional alguna:

er kneift mir/mich in den Arm,

* er kneift für mich in den Arm,

er küsst mir/mich auf die Hand,

* er küsst auf meine Hand.

Aquí debemos hacer referencia también a lo que muchos autores denominan Trägerdativ, que lo podemos definir como un tipo de possessiver Dativ, pero con una referencia semántica a prendas de vestir y objetos. Obsérvense los siguientes ejemplos:

ich ziehe mir den Mantel an,

mir rutscht die Hose,

das Wasser läuft mir in die Sohle,

sich eine Uhr anlegen.

Del mismo modo que con el possessiver Dativ, en este tipo de dativo no se da ningún tipo de concurrencia con frase preposicional alguna:

*den Mantel ziehe ich für mich,

*die Hose rutscht für mich.

En último lugar, el dativo ético o ETISCHER DATIV, y para ello observemos los siguientes ejemplos:

Verachte mir meinen Lehrer nicht!, mach mir meine Tür nicht kaputt!

schlag mir meinen Bruder nicht! 
Oraciones de este tipo han quedado lexicalizadas en la lengua alemana actual y en este sentido afirma Hans glinz que este tipo de dativo es el más relevante de todos, siendo el único a destacar respecto al dativo obligatorio o eigentliches Dativobjekt ${ }^{15}$.

El dativo ético o ethischer Dativ se caracteriza no sólo por tener un especial carácter facultativo en la oración, sino por tener la única función de enfatizar la persona a la que va referida la acción. Es, por lo tanto, el único caso de dativo en el que se admite una concurrencia de una misma persona tanto en el adjetivo posesivo como en la forma de dativo.

De este modo, construcciones como

ich bin mir bewußt,

ich bin mir sicher,

se pueden considerar ejemplos de dativo ético. En ellas, como se observa, concurren dos elementos -sujeto en nominativo y objeto indirecto en dativo- con referencia a una misma persona. Por este caráter enfatizador del dativo ético hẻmos de destacar que nunca se puede situar en el primer lugar de la oración y en este sentido lo definen muchos autores como nicht eststellenfähig. De este modo, nunca encontraremos construcciones como

*mir bin ich bewußt,

*mir verachte meinen Lehrer nicht!

En los otros casos de dativo sí es posible encontrar el dativo como primer elemento de la oración o, lo que es lo mismo, ocupando el denominado Vorfeld y enfatizando de este modo la persona a la que va referida la acción verbal. Obsérvese:

mir habe ich die Uhr angelegt!

ihm hat er zwei Zähne ausgeschlagen!

Debemos destacar también el carácter coloquial del dativo ético, es decir, normalmente encontraríamos la construcción

mach meine Tür nicht kaputt!

pero en el lenguaje coloquial es donde puede aparecer con cierta frecuencia la construcción con dativo ético:

mach mir meine Tür nicht kaputt!

Como conclusión podemos afirmar que la lengua alemana actual ha sufrido una pérdida en el uso dativo en favor de otras construccciones con las que en la mayoría de los casos aún concurre. Heide Wegener apoya también esta teoría haciendo además una especial referencia a una primitiva función causativa del dativo ${ }^{16}$. Según él dicho caso se ha visto en este sentido

15 Glinz, Hans. Deutsche Grammatik. Athenäum. Frankfurt. 1971. Pg. 58

16 Wegener, Heide. Kasus im Deutschen, Japanischen und anderen Sprachen-am Beispiel des Dativs. En: Akten des VIII. Internationalen Germanisten-Kongresses. Tokyo 1990. Iudicium Verlag. München. 1991. Pg. 96. 
completamente sustituído por el acusativo a lo largo del último siglo. Para ello recoge ejemplos como

*er läßt mir aber nichts merken,

Estas construcciones están hoy en desuso debido a la concurrencia que estas oraciones tenían en principio con la fórmula de acusativo

er läßt mich aber nichts merken, wenn Sie mich wissen lassen.

y a la completa sustitución que éstas últimas han hecho respecto a las primeras. 